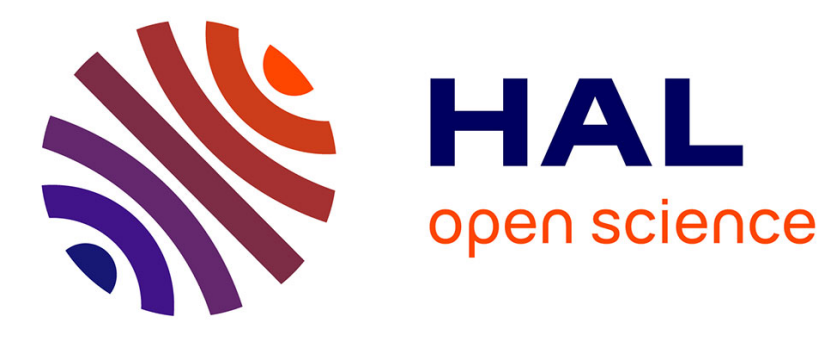

\title{
Brain Peroxisomes
}

Doriane Trompier, A. Vejux, A. Zarrouk, Catherine Gondcaille, Flore Geillon, T. Nury, Stéphane Savary, Gérard Lizard

\section{- To cite this version:}

Doriane Trompier, A. Vejux, A. Zarrouk, Catherine Gondcaille, Flore Geillon, et al.. Brain Peroxisomes. Biochimie, 2013, 98, pp.102-110. 10.1016/j.biochi.2013.09.009 . hal-01185043

\section{HAL Id: hal-01185043 \\ https://hal.science/hal-01185043}

Submitted on 18 Aug 2015

HAL is a multi-disciplinary open access archive for the deposit and dissemination of scientific research documents, whether they are published or not. The documents may come from teaching and research institutions in France or abroad, or from public or private research centers.
L'archive ouverte pluridisciplinaire HAL, est destinée au dépôt et à la diffusion de documents scientifiques de niveau recherche, publiés ou non, émanant des établissements d'enseignement et de recherche français ou étrangers, des laboratoires publics ou privés. 
Mini-review

\title{
Brain peroxisomes
}

\author{
D. Trompier ${ }^{\text {a }}$, A. Vejux ${ }^{\text {a }}$, A. Zarrouk ${ }^{\text {a, b }}$, C. Gondcaille ${ }^{\text {a }}$, F. Geillon ${ }^{\text {a }}$, T. Nury ${ }^{\text {a }}$, S. Savary ${ }^{\text {a }}$, \\ G. Lizard ${ }^{\mathrm{a}}$ * \\ ${ }^{a}$ Université de Bourgogne, Laboratoire «Bio-PeroxIL» de Biochimie du Peroxysome, Inflammation et Métabolisme Lipidique (EA7270)/INSERM, 6 Bd Gabriel, \\ Dijon F-21000, France \\ b Université de Monastir, Faculté de Médecine, LR12ES05, Lab-NAFS 'Nutrition - Functional Food E’ Vascular Health', Avenue Avicenne, Monastir, Tunisia
}

\section{A R T I C L E I N F O}

\section{Article history:}

Received 31 May 2013

Accepted 12 September 2013

Available online 21 September 2013

\section{Keywords:}

Peroxisome disorders

Demyelination

Oxidative stress

Inflammation

Alzheimer's disease

\begin{abstract}
A B S T R A C T
Peroxisomes are essential organelles in higher eukaryotes as they play a major role in numerous metabolic pathways and redox homeostasis. Some peroxisomal abnormalities, which are often not compatible with life or normal development, were identified in severe demyelinating and neurodegenerative brain diseases. The metabolic roles of peroxisomes, especially in the brain, are described and human brain peroxisomal disorders resulting from a peroxisome biogenesis or a single peroxisomal enzyme defect are listed. The brain abnormalities encountered in these disorders (demyelination, oxidative stress, inflammation, cell death, neuronal migration, differentiation) are described and their pathogenesis are discussed. Finally, the contribution of peroxisomal dysfunctions to the alterations of brain functions during aging and to the development of Alzheimer's disease is considered.
\end{abstract}

(c) 2013 Elsevier Masson SAS. All rights reserved.
Peroxisomes are DNA free organelles present in nearly all eukaryotic cells (with the exception of erythrocytes) including unicellular eukaryotes and higher plant cells [1]. They are morphologically characterized by a single limiting membrane and a finely granular matrix with a range in size from 0.1 to $1 \mu \mathrm{m}$ in diameters (Fig. 1). However, peroxisome shape, size, and cellular content show considerable variations in response to fission, fusion, and proliferation mechanisms. Among the different organs, peroxisomes are numerous in liver (hepatocytes) and peroxisomal proteins expression has been detected in different areas of the brain (especially cerebellum, hippocampus) and in all neural cell types with a preponderance for glial cells (oligodendrocytes, astrocytes) [2]. In the cytoplasm, peroxisomes are highly dynamic organelles with marked plasticity that have been

Abbreviations: ABC, ATP-Binding Cassette; ACOX, acyl-CoA oxidase; AD, Alzheimer's disease; AMACR, $\alpha$-methyl-acyl-CoA-racemase; RD, Refsum disease; $A \beta$, amyloid $\beta$ peptide; DBPD, D-bifunctional protein deficiency; DHA, docosahexaenoic acid; DHAP, dihydroxyacetone phosphate; DHCA, dihydroxycholestanoic acid; IRD, infantile Refsum disease; MUFA, monounsaturated fatty acid; NALD, neonatal adrenoleukodystrophy; NO, nitric oxide; PBD, peroxisome biogenesis disorders; PEX, peroxin; PNALD, pseudo-neonatal adrenoleukodystrophy; PUFA, polyunsaturated fatty acid; RCDP, rhizomelic chondrodysplasia punctata; RNS, reactive nitrogen species; ROS, reactive oxygen species; THCA, trihydroxycholestanoic acid; VLCFA, very-long-chain fatty acid; X-ALD, X-linked adrenoleukodystrophy; ZSS, Zellweger syndrome spectrum; ZS, Zellweger syndrome.

* Corresponding author. Tel.: +33 (0)380396256; fax: +33 (0)380396250.

E-mail address: gerard.lizard@u-bourgogne.fr (G. Lizard). shown to move in a motor protein-dependent manner along microtubules in mammals [3] or actin filaments in plants and fungi [4]. As peroxisomes lack DNA, most of the peroxisomal membrane and matrix proteins are synthesized on free polyribosomes in the cytoplasm and are then transported posttranslationally to the organelle. So, specific proteins import pathways are required. They include two classes of matrix targeting signals for peroxisomal proteins [PTS1 (Peroxisomal Targeting Signal 1) and PTS2] which are recognized by cytosolic receptors [PEX5 (peroxin 5) and PEX7, respectively] escorting their cargo proteins to, or possibly across, the peroxisome membrane.

The crucial roles of peroxisomes in human health became obvious when some peroxisomal abnormalities, which are often not compatible with life or normal development, were identified in severe neurodegenerative and demyelinating brain diseases. This review describes the metabolic role of peroxisomes with a special focus on the brain and lists the human brain peroxisomal disorders resulting from a peroxisome biogenesis defect or from a single peroxisomal enzyme defect. We focused on the abnormalities of myelination, oxidative stress, inflammation, cell death and neuronal migration problems observed in brain peroxisomal disorders and discussed the causes of these abnormalities. Finally, the potential role of peroxisomes in brain degeneration is considered with the involvement of peroxisomes in cell aging and in the development of Alzheimer's disease. 

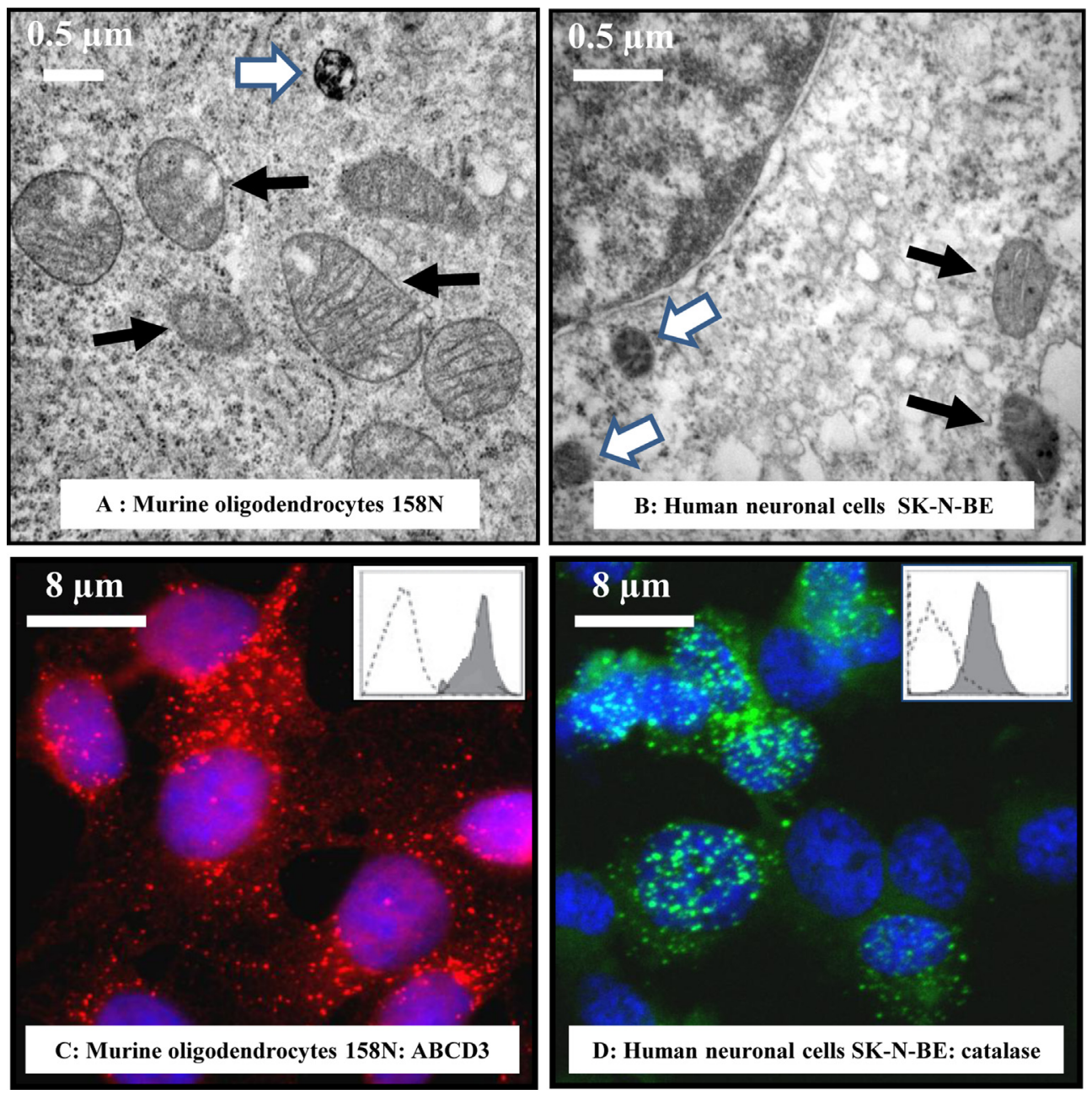

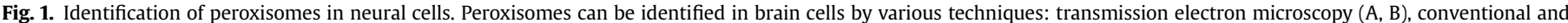

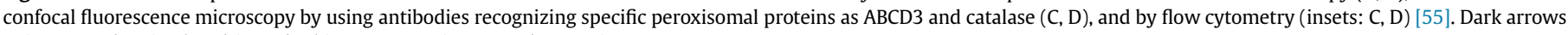
point towards mitochondria and white arrows point towards peroxisomes.

\section{Metabolic role of peroxisomes in brain}

More than 50 enzymes participating in various metabolic pathways, especially lipid and oxidative metabolic pathways, have been identified [5]. The main peroxisomal functions concern lipid metabolism with the $\beta$-oxidation of diverse compounds and the $\alpha$-oxidation of phytanic acid [6]. Peroxisomes are also crucial for hydrogen peroxide detoxification, via catalase activity and are also involved in the degradation of purines, D-amino acids, polyamines, eicosanoids and uric acid (in non-primates). In brain, one of the main roles of peroxisomes is to degrade saturated very-long-chain fatty acids (VLCFA) like C24:0 and C26:0 and to participate in the equilibrium of these compounds particularly enriched in the myelin. Peroxisomal $\beta$-oxidation of VLCFA consists in 4 enzymatic steps: dehydrogenation, hydration, dehydrogenation again, thiolytic cleavage releasing a shortened fatty acid and acetyl-CoA [6]. The released fatty acid undergoes subsequent rounds of $\beta$-oxidation till the formation of octanoyl-CoA. Carnitine octanoyltransferase catalyzing the exchange of acyl groups between carnitine and coenzyme A ( $\mathrm{CoA})$ contributes to connect peroxisomes with mitochondria, octanoyl-carnitine being transported to the mitochondria to be fully degraded. Peroxisomal $\beta$-oxidation also concerns monounsaturated (MUFA), polyunsaturated fatty acids (PUFA) and their derivatives (eicosanoids and docosanoids), 2-methyl branched-chain fatty acids and dicarboxilic acids whose excesses can be as toxic as the accumulation of saturated VLCFA [6]. Degradation of eicosanoids is of particular importance given the inflammatory pathways that depend on these molecules. Arachidonic (C20:4 n-6) and eicosapentaenoic (C20:5 n-3) acids, and their derivatives (prostaglandins, leukotrienes, prostacyclins, thromboxanes) are the known mediators of a broad range of inflammatory reactions [7].

Peroxisomal $\beta$-oxidation may not be considered as a simple catabolic pathway since the last step of the synthesis of docosahexaenoic acid (DHA, C22:6 n-3) involves one turn of $\beta$-oxidation from C24:6 n-3 [8]. DHA is the best represented PUFA in brain and nervous tissues. In addition to its structural role in membrane lipids, DHA has been shown to act at different levels in brain for neurotransmission, synaptic plasticity, gene expression, calcium concentration homeostasis [7,9]. Its enzymatic conversion to resolvins, neuroprotectins and maresins provides very active antiinflammatory molecules that can inhibit the generation of prostaglandins, leukotrienes, and thromboxanes $[10,11]$. The brain concentration of DHA depends on both dietary content in n-3 PUFA and liver synthesis [12]. Another important function of peroxisomes in the brain concerns the synthesis of plasmalogens since the first 2 steps of this anabolic pathway take place in peroxisomes [13]. After the acylation of dihydroxyacetone phosphate, which forms a 1-acyl-DHAP, the acyl group is replaced by a fatty alcohol synthetized within peroxisomes from acyl-CoA [14]. The 1-alkyl-DHAP is 
then reduced to yield a 1-alkyl-phosphoglycerol which is further processed in the endoplasmic reticulum. The major predicted roles of plasmalogens are to contribute to membrane fluidity, to buffer oxidative stress and to serve as reservoirs for second messengers [15].

Beyond their role in lipid metabolism, peroxisomal functions are involved in the control of oxidative stress homeostasis [5]. Peroxisomal enzymes such as acyl-CoA oxidases (ACOX) or inducible nitric oxide synthase (iNOS) [16] are known to generate oxidative stress with reactive oxygen species (ROS) and reactive nitrogen species (RNS). ROS molecules, especially superoxide anions $\left(\mathrm{O}_{2}{ }^{-{ }^{-}}\right)$ and hydrogen peroxide $\left(\mathrm{H}_{2} \mathrm{O}_{2}\right)$, and RNS molecules with nitric oxide (NO), are produced in part in peroxisomes [17]. In the same time, peroxisomes possess enzymatic components which counteract the oxidative and nitrosative stress. These enzymes include catalase, superoxide dismutase 1, or glutathione S-transferase [17].

\section{Human brain peroxisome disorders}

Peroxisomes contribute to central metabolic pathways in the brain and their functional importance in human health is highlighted by peroxisomal disorders. Currently, these disorders are classified into two groups: i) peroxisome biogenesis disorders (PBDs) and ii) single peroxisomal enzyme deficiencies (Table 1).

\subsection{Peroxisome biogenesis disorders}

The PBDs result from a failure in organelle formation leading to multiple metabolic abnormalities. The PBDs can be divided into two subtypes; i) the Zellweger syndrome spectrum (ZSS) disorders and ii) the rhizomelic chondrodysplasia punctata (RCDP) type 1 .

The ZSS is a group of inherited diseases which consists of at least three different but overlapping clinical phenotypes with variable severity of neurologic symptoms [18]. The Zellweger syndrome (ZS) is the most severe form, the neonatal adrenoleukodystrophy (NALD) is intermediate and the infantile Refsum disease (IRD) is the less severe phenotype. PBDs are autosomal recessive disorders all caused by a defect in PEX genes which encode for peroxins, proteins necessary for peroxisome biogenesis and involved in the import process of peroxisomal integral membrane and matrix proteins. It is not possible to predict the defective PEX gene from the biochemical or clinical phenotype. However, the type of mutation of the defective PEX gene correlates with the clinical severity and with the impact on peroxisome function and assembly. Biochemical abnormalities are less severe in patients with milder form of PBDs whose skin fibroblasts contain residual peroxisome activity. Cells from patients with severe ZS are devoid of peroxisomes.

RCDP type 1 is clinically clearly distinct from the ZSS disorders. Patients with RCDP type 1 have mutations in PEX7 gene which encodes a cytosolic receptor that directs PTS2 peroxisomal matrix enzymes to the peroxisomes. Three PTS2 enzymes are transported by PEX7: the ACAA1 thiolase of the $\beta$-oxidation pathway, the phytanoyl-CoA hydroxylase of the $\alpha$-oxidation pathway, and the alkyl-DHAP synthase involved in the synthesis of plasmalogens. The function of ACCA1 can be taken over by a second thiolase (SCPx) transported to the peroxisomes by PEX5, explaining why plasma VLCFA levels are normal in RCDP patients. However, defective targeting of the alkyl-DHAP synthase and the phytanoylCoA hydroxylase leads to plasmalogen deficiency and also to phytanic acid accumulation [19].

\subsection{Single peroxisomal enzyme deficiencies}

The second category of peroxisomal disorders includes disorders which result from the deficiency of a single peroxisomal enzyme involved in $\beta$-oxidation, $\alpha$-oxidation or ether lipid synthesis.

Four main defined disorders of peroxisomal $\beta$-oxidation have been identified; i) X-linked adrenoleukodystrophy (X-ALD), ii) acylCoA oxidase 1 deficiency (or PNALD; pseudo-neonatal adrenoleukodystrophy), iii) D-bifunctional protein deficiency (DBPD) and iv) $\alpha$-methyl-acyl-CoA-racemase (AMACR) deficiency. X-ALD is the most common peroxisomal disorder with impaired $\beta$-oxidation of saturated VLCFA resulting in the accumulation of VLCFA in plasma and tissues, which is a reliable diagnostic marker of the disease. In $\mathrm{X}$-ALD brain, increased VLCFA levels are found especially in the cholesterol ester fraction but also in cerebrosides, phosphatidylcholine, sphingomyelin and sulfatides [20]. This complex neurodegenerative disorder is characterized by a huge clinical variability both in the age of onset and in symptoms. The responsible gene for $\mathrm{X}-\mathrm{ALD}$ is the $A B C D 1$ gene which encodes for a peroxisomal ATPBinding Cassette $(A B C)$ transporter involved in the transport of VLCFA-CoA into the peroxisomes for their $\beta$-oxidation [21,22]. The clinical presentation of PNALD and DBPD resembles that of the disorders of peroxisome biogenesis although there are defects in only one single enzymatic process. ACOX1 catalyzes the $\alpha, \beta-$ dehydrogenation of a range of acyl-CoA esters among which saturated VLCFA, resulting in elevated VLCFA levels in plasma of patients although peroxisomes were present and even of enlarged sizes. The D-bifunctional protein deficiency causes abnormal oxidation of VLCFA, pristanic acid and di- and trihydroxycholestanoic acids (DHCA, THCA) resulting in their accumulation in plasma of patients. The AMACR deficiency is a rare autosomal recessive disorder that was reported in ten adults and four infants worldwide. The enzyme catalyzes the interconversion of (2R)- and (2S)-stereoisomers of $\alpha$-methyl branched-chain fatty acids (such as pristanic acid), DHCA and THCA, which explains the accumulation of pristanic acid and bile-acid intermediates in patients.

The absence of the first enzyme involved in the $\alpha$-oxidation pathway, the phytanoyl-CoA hydroxylase, is responsible for Refsum disease (RD). All patients accumulate phytanic acid in blood and tissues. Rhizomelic chondrodysplasia punctata (RCDP) of type 2 and of type 3 are lethal disorders, respectively associated with mutation in the GNPAT and AGPS genes that encode for the two enzymes (DHAP acyltransferase and alkyl DHAP synthase) catalyzing the first steps of ether lipid biosynthesis in peroxisomes. The clinical phenotypes are indistinguishable from that of patients with RCDP of type 1 and these disorders are characterized by decreased levels of plasmalogens.

\section{Physiopathological consequences of peroxisomal dysfunctions}

\subsection{Differentiation and neuronal migration abnormalities}

Abnormalities in neuronal migration are classically seen in ZS, NALD and DBPD patients [23]. The neuronal migration defects are less prominent in NALD patients and often indistinguishable between ZS and DBPD patients. Only few cases among IRD and RCDP patients have displayed cerebral neuronal migration problems [23]. ZS neuropathological features include impaired brain neuronal migration leading to characteristic cytoarchitectonic abnormalities in the cerebral cortex and in the cerebellum [24]. The malformation of the cerebral cortex is most severe, encountered postnatally and on fetuses, and results from an impaired migration of neuroblasts to form the cerebral cortical plate. The cerebellum contains heterotopic Purkinje cells in the white matter or combinations of abnormally arranged Purkinje cells and granule neurons. In addition, defects in neuronal differentiation, proliferation and survival may also contribute to the malformations, as assessed by in vivo 
Table 1

Human brain peroxisome deficiencies and their pathologies. A plus sign $(+)$ denotes presence; absence of sign means either there is no defect or that the defect has not been described (yet); $a+/-$ sign denotes that the symptom may or may not be present. For biochemical features, the presence $(+)$ of increased and decreased levels of fatty acids/lipids are indicated.

\begin{tabular}{|c|c|c|c|c|c|c|c|c|c|c|c|}
\hline \multirow[t]{3}{*}{ Disease } & \multicolumn{4}{|c|}{ Peroxisome biogenesis disorders (PBD) } & \multicolumn{7}{|c|}{ Single peroxisomal enzyme deficiency } \\
\hline & \multicolumn{3}{|c|}{ Zellweger syndrome spectrum } & \multirow{2}{*}{$\begin{array}{l}\text { RCDP } \\
\text { type } 1\end{array}$} & \multirow[t]{2}{*}{$\mathrm{X}-\mathrm{ALD}$} & \multirow[t]{2}{*}{ PNALD } & \multirow{2}{*}{$\begin{array}{l}\text { DBP } \\
\text { deficiency }\end{array}$} & \multirow{2}{*}{$\begin{array}{l}\text { AMACR } \\
\text { deficiency }\end{array}$} & \multirow{2}{*}{$\begin{array}{l}\text { Refsum } \\
\text { disease }\end{array}$} & \multirow[t]{2}{*}{ RCDP type 2} & \multirow[t]{2}{*}{ RCDP type 3} \\
\hline & $\overline{\mathrm{ZS}}$ & NALD & IRD & & & & & & & & \\
\hline OMIM & & & & 215100 & 300100 & 264470 & 261515 & 614307 & 266500 & 222765 & 600121 \\
\hline Gene defect & $\begin{array}{l}\text { PEX 1,2,3,5,6,10,12, } \\
13,14,16,19 \text { or } 26\end{array}$ & $\begin{array}{l}\text { PEX } 1,5,6,10 \\
12,13, \text { or } 26\end{array}$ & $\begin{array}{l}\text { PEX } 1,2,12 \\
\text { or } 26\end{array}$ & PEX 7 & $A B C D 1$ & ACOX1 & HSD17B4 & $A M A C R$ & PHYH & GNPAT & AGPS \\
\hline Peroxisomes & Absence (or "ghost") & $\begin{array}{l}\text { Absence } \\
\text { (or "ghost") }\end{array}$ & $\begin{array}{l}\text { Absence } \\
\text { (or "ghost") }\end{array}$ & + & + & $+($ abnormal) & $+($ abnormal) & + & + & + & + \\
\hline \multicolumn{12}{|l|}{ Clinical features } \\
\hline Hypotonia & + & + & + & + & & + & + & & & + & + \\
\hline Craniofacial dysmorphy & + & + & + & + & & + & + & & & + & + \\
\hline Skeletal defect & + & + & + & + & & + & + & & + & + & + \\
\hline Growth retardation & + & + & + & + & & + & + & & & + & + \\
\hline Mental retardation & + & + & + & + & $+1-$ & + & + & $+1-$ & & + & + \\
\hline Spasticity & + & + & + & + & + & + & + & + & & + & + \\
\hline Seizure & + & + & + & + & + & + & + & + & & + & + \\
\hline Retinopathy/vision failure & + & + & + & + & + & + & & + & + & + & + \\
\hline $\begin{array}{l}\text { Hearing failure } \\
\text { Anosmia }\end{array}$ & + & + & + & & + & + & & & $\begin{array}{l}+ \\
+\end{array}$ & + & + \\
\hline \multirow{2}{*}{\multicolumn{12}{|c|}{ Neurological abnormalities }} \\
\hline & & & & & & & & & & & \\
\hline Cortex malformation & + & + & + & + & & + & + & + & & & \\
\hline Neuronal migration defect & + & + & + & + & & & + & & & & \\
\hline Cerebellum malformation & + & + & + & + & & & + & + & + & & \\
\hline \multicolumn{12}{|l|}{ Brain degeneration } \\
\hline Leukodystrophy & + & + & + & & + & + & + & $+1-$ & & & \\
\hline Peripheral neuropathy & + & + & + & & $+1-$ & + & + & + & + & & \\
\hline \multicolumn{12}{|l|}{ Biochemical features } \\
\hline Ether lipids (plasmalogens) $\downarrow$ & + & + & + & + & + & & & & & + & + \\
\hline DHA $\downarrow$ & + & + & + & + & & + & & & & & \\
\hline VLCFA $\uparrow$ & + & + & + & & + & + & + & & & & \\
\hline Branched-chain fatty acids $\uparrow$ & + & + & + & + & & + & + & + & + & & \\
\hline \multirow[t]{2}{*}{ Bile-acid intermediates $\uparrow$} & + & + & + & & & & + & + & & & \\
\hline & \multicolumn{4}{|c|}{ Multiple impaired metabolic pathways } & \multicolumn{3}{|c|}{ Impaired $\beta$-oxidation } & & $\begin{array}{l}\text { Impaired } \\
\alpha \text {-oxidation }\end{array}$ & \multicolumn{2}{|c|}{$\begin{array}{l}\text { Impaired ether lipid } \\
\text { biosynthesis }\end{array}$} \\
\hline Survival (-year-old) & $<1$ & $<10$ & Up to adulthood & $<10$ & $<10$ & $<5$ & $<2$ & & $\begin{array}{l}\text { Normal } \\
\text { (under diet) }\end{array}$ & $<10$ & $<10$ \\
\hline Approximative onset & Newborn ${ }^{\mathrm{a}}$ & Newborn $^{\mathrm{a}}$ & Newborn $^{\mathrm{a}}$ & Newborn $^{a}$ & Childhood & Newborn $^{\mathrm{a}}$ & Newborn $^{\mathrm{a}}$ & Adulthood & Childhood & Childhood $^{\mathrm{b}}$ & Childhood $^{\mathrm{b}}$ \\
\hline
\end{tabular}

Abbreviations: ZS, Zellweger syndrome; NALD, neonatal adrenoleukodystrophy; IRD, infantile Refsum disease; RCDP, rhizomelic chondrodysplasia punctata; X-ALD, X-linked adrenoleukodystrophy; PNALD, pseudo-neonatal adrenoleukodystrophy; DBP, D-bifunctional protein; AMACR, $\alpha$-methyl-acyl-CoA-racemase; PEX, peroxin; ABCD1, ATP-Binding Cassette D1; ACOX, acyl-CoA oxidase; PHYH, phytanoyl-CoA hydroxylase; GNPAT, glyceronephosphate O-acyltransferase; AGPS, alkylglycerone-phosphate synthase; DHA, docosahexaenoic acid; VLCFA, very-long-chain fatty acid.

${ }^{a}$ In patients affected at birth, the onset is actually already during intrauterine development.

b RCDP type 2 and 3 can start as early as RCDP type 1 . 
studies in Pex2 $2^{-I-}$ Zellweger mice [24]. The neuronal migration abnormalities in DBPD are similar to those in ZS [25,26] and more extensive than the abnormalities seen in NALD [27]. The precise molecular mechanisms causing the neuronal migration and differentiation defects remains obscure. The peroxisomal metabolism in brain as well as in extraneuronal tissues influence the neuronal migration process in Pex 5 knock-out mice, a model for Zellweger syndrome [28]. It seems unlikely that VLCFA accumulation, plasmalogens depletion or DHA deficiency by themselves cause the migration defect in peroxisomal disorders but they may rather contribute to the defects.

\subsection{Abnormal myelination}

In humans, myelin production begins in the fifth month of fetal development in the motor roots of peripheral nervous system, and is followed by myelination of the spinal cord and the brain. Although normal myelination occurs mainly in the first two years of life, myelination continues for 20-30 years in the human cerebral white matter. Myelin defects in brain peroxisomal disorders include delayed myelination (with increasing content of myelin over time), hypomyelination (with unchanged lack of myelin over time), demyelination (degenerative loss of myelin) and dysmyelination (abnormally formed myelin). The clear distinction between demyelination and abnormal myelination is often rendered difficult before the first two years of life when the two processes can occur simultaneously [29].

Within the ZSS patients displaying a mild and severe phenotype, the disturbed function of peroxisomes is associated with brain white matter abnormalities that can be demyelinative and/or dysmyelinative [29]. All ZS patients have impaired myelination which appears to be primarily a hypomyelination [30]. In NALD patients, the progressive demyelination seen is diffuse and affects cerebral and cerebellar hemispheres. In IRD patients, myelination is abnormal, but there is no active demyelination. White matter may be morphologically normal in ZSS patients surviving the first year [31]. The severity of myelin abnormalities is correlated with the plasmalogen level in RCDP spectrum [32]. Indeed, no myelin abnormalities were seen in the patients with mild phenotype, and delay in supratentorial white matter myelination was observed in patients with the severe phenotype of both RCDP type 1 and 3. In DBPD patients, magnetic resonance imaging studies may show cerebral and cerebellar demyelination [33]. While demyelination in the peripheral nervous system is documented in Refsum disease patients, no myelin abnormalities have been evidence in the brain [29]. X-ALD is a severe demyelinating disease characterized by a progressive inflammatory demyelination in the brain, the progression being slower in the adolescent and adult forms compared to the childhood form [34]. The most frequent pattern is the characteristic symmetrical demyelination that occurs in the parietooccipital region.

The lack of myelination seen in PBD and RCDP type 2 and 3 patients may result from the depletion of plasmalogen levels (major component of the normal myelin membrane). Abnormal myelination may also be due to VLCFA accumulation in membrane lipids. VLCFA are particularly enriched in myelin along with other specific lipids $[35,36]$. VLCFA may contribute to myelin stability and insulating properties. However, their excess is thought to physically destabilize myelin sheath $[23,37]$ and/or become toxic for nervous cells in charge of myelin synthesis (oligodendrocytes) and brain survey (microglia) [38,39]. VLCFA accumulation is more pronounced in the center of X-ALD demyelination plaques, and is therefore suspected to initiate the destabilization of the myelin organization leading to cerebral demyelination [34]. Peroxisomes are found in all neural cell types and their abundance decreases from birth to adulthood. Alteration of peroxisomal functions in oligodendrocytes appears to be the main pathogenic factor leading to demyelination [34]. However, alteration of peroxisomal functions in other brain cell types may contribute to aggravate the pathological phenotype [40].

\subsection{Inflammation}

The part taken by peroxisomes in cytokinic inflammation was established in the 90s, on brain lesions of patients with X-ALD which revealed increased expression of pro-inflammatory cytokines (IL-1, TNF- $\alpha$ ) and ICAMs (Intercellular Adhesion Molecules) by astrocytes and microglial cells [41-43]. To understand the role of inflammation in X-ALD, the expression of inflammatory cytokines in different regions of brain with the childhood form has been investigated [44]. An increase of mRNA expression of different cytokines (IL- $1 \alpha$, IL-2, IL-3, IL-6, TNF- $\alpha$, GM-CSF), chemokines (CCL2, $-4,-7,-11,-21,-22$, CXCL1, CX3CL1 and SDF-2) and iNOS was observed in the active edge of demyelinating plaques of the brain compared to normal looking area [44]. In addition, a spatial relationship between VLCFA accumulation and inflammation has been evidenced [44]. Similar observations were obtained in mutant mice where Pex5 is selectively inactivated in oligodendrocytes with neuroinflammation characterized by an increase of proinflammatory (TNF- $\alpha$, IFN- $\gamma$ ) and anti-inflammatory (IL-10) factors, and chemoattractants for lymphomononuclear cells (MIP- $1 \alpha$, MCP-1, MCP-5 and IP-10) [45]. In the mouse model with neural peroxisome dysfunction (Nestin-Pex5 knock-out mice) or in Pex1 mutant Drosophila, an induction of innate immunity was observed in brain with up regulation of pathways related to immune responses: transendothelial migration, antigen processing and presentation (C1q, subcomponent of classical complement system, is up-regulated in corpus callosum), cytokine-cytokine receptor interaction and toll-like receptor signaling pathways (TNF- $\alpha$, TLR2 and Cxcl-1 up-regulated in corpus callosum before demyelination) $[46,47]$. In mice with peroxisome deficiency in neural cells (Nestin$P e x 5^{-I-}$ ), the innate immune system is early activated (3 weeks after birth), with strong and persistent inflammatory response [46]. Noteworthy, in X-ALD patients, a relationship between inflammation and oxidative stress has been suggested [48]. Indeed, some redox sensors can be present in key regulators of inflammation, for example NF- $\kappa$ B, which has redox-active cysteine residues, Nrf2. It was proposed that high levels of ROS release by neural cells (glial cells, neurons) may favor an inflammatory response [48].

Some studies also support that non cytokinic inflammation also depends on peroxisomal activity since 5-lipoxygenase (5-LOX) and cycloxygenase-2 (COX-2) are induced in Abcd1/Abcd2-silenced astrocytes [49]. Moreover, 5-LOX-derived leukotrienes were significantly elevated in all the areas of white matter in the brain of the cerebral form of X-ALD. Unlike COX-2 expression, which was moderately high only in the plaque area, expression of 5-LOX and cytosolic phospholipase A2 was prominent in all the areas [49].

In the three hit hypothesis it is suggested that oxidative stress triggers neuroinflammation contributing to cell death and demyelination [50].

\section{Consequences of peroxisomal dysfunction at the cellular level}

\subsection{Oxidative stress}

The disturbance of redox homeostasis is thought to initiate and/ or amplify the progression of neurological diseases. Alterations of peroxisomal functions contribute by themselves to this stress but they have also consequences on the mitochondrial redox status 
[51]. These consequences on mitochondria are apparently not restricted to oxidative stress but would be also associated with bioenergetic and metabolic failure [48]. Oxidative damages have been documented in human X-ALD brain tissue with the childhood form: increase mRNA expression of iNOS [44] and presence of lipid peroxidation products (4-hydroxynonenal and malonedialdehyde) [52]. Oxidative stress can also be observed in the case of deletion of a single allele of the Pex11 $\beta$ gene in mice; this deletion in mice leads to oxidative damage of the nucleic acid and to an increase of manganese superoxide dismutase in cerebellum and neocortex [53]. In Pex11 $\beta^{-1-}$ mice, a decrease in catalase is observed in neocortex [53]. In a mouse model of Zellweger syndrome (Pex13 null mice), mitochondria-mediated oxidative stress in cultured cerebellar neurons and in cerebellum sections was also reported [54]. On murine oligodendrocyte $158 \mathrm{~N}$ cells, by using siRNAs raised against Abcd1 and Acox1, an increased overproduction of ROS and RNS potentiated by VLCFA was also observed [38,55]. Gene silencing leads to lipid peroxidation, enhanced accumulation of lipids, and induction of cell death.

\subsection{Cell death}

In pathologies involving peroxisome dysfunction in brain, VLCFA accumulation, oxidative stress and inflammation are associated with cell death. The type of cell death is not well characterized: necrosis or apoptosis are mentioned. Apoptosis (DNA ladder pattern and terminal deoxynucleotidyl transferase staining) associated with high level of ceramide and low level of GSH has been described in X-ALD brain [56]. In the brain of X-ALD patients with the cerebral phenotype, apoptosis mainly concerns oligodendrocytes with presence of activated-caspase- 3 and fragmented DNA [57]. Apoptosis is observed in brain areas with microglial activation and can represent an early stage in lesion evolution in X-ALD disease [58]. However, Ito et al. [59] suggest the major mode of oligodendrocytic death in the brain of X-ALD patients is cytolysis, rather than apoptosis.

In a model of Zellweger syndrome (Pex-13 null mice), neuronal cell death and mitochondria-mediated oxidative stress have been evidenced in cerebellum sections and in cultured cerebellar neurons [54]. Both in primary neuronal cultures from the neocortex and cerebellum of Pex $11 \beta^{+/-}$and Pex $11 \beta^{-1-}$ mice and in the brain of these mice, neuronal cell death with typical characteristics of apoptosis is observed: caspase-3 activation, membrane leakage [53].

Several in vitro studies provided more information about the processes involved in cell death induced by C22:0 and VLCFA accumulation (C24:0, C26:0) on on mixed primary culture of glial and neural cells [39]. This cell death is characterized by mitochondrial dysfunction and $\mathrm{Ca}^{2+}$ perturbation [39]. In murine $158 \mathrm{~N}$ oligodendrocyte cells treated with VLCFA (C24:0; C26:0), no sign of apoptosis was observed [60] but mitochondrial depolarization associated with an overproduction of superoxide anions and a loss of lysosomal membrane integrity have been described [55]. Similar results were observed on human neuronal cells SK-N-BE treated with C22:0, C24:0 or C26:0 [61].

\section{Potential role of peroxisomes in brain degeneration}

\subsection{Peroxisomes and aging}

The implication of peroxisomes in cell aging and in the development and progression of specific age-related degenerative diseases is supported by the dysregulation of ROS and RNS- production occurring in these organelles [62].

Human patients suffering from an inherited deficiency of catalase (hypocatalasemia), face an increased risk of developing age-related diseases including diabetes, atherosclerosis, and cancer [63]. Furthermore, cells from hypocatalasemic patients accumulate hydrogen peroxide and harbor signs of age-associated pathologies [64]. In hypocatalasemic conditions, the ability of peroxisomes to import proteins, to regulate its growth and division and to process ROS is altered [65]. Moreover, catalase is suspected to play role in lifespan since longevity is positively impacted by reduced cellular oxidative stress [62]. When cells enter senescence (described as a loss of the ability of metabolically active cells to replicate), catalase is translocated from the peroxisomes to the cytosol. Since restoration of catalase into the peroxisomes slows down senescence, catalase may represent a

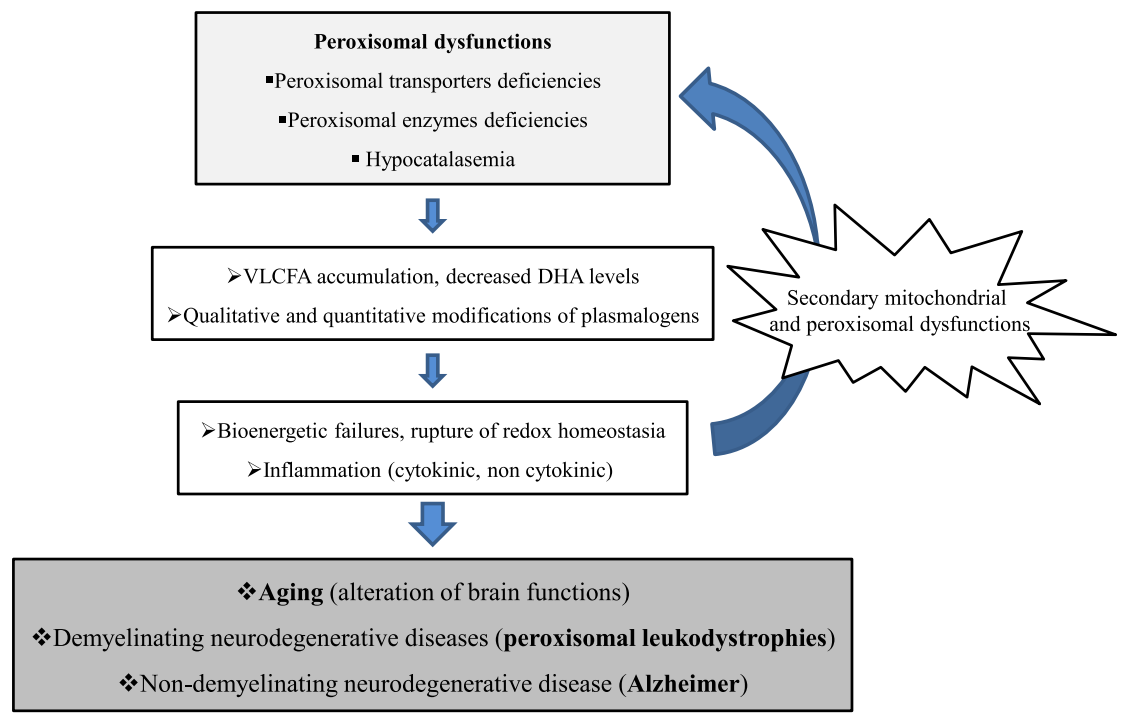

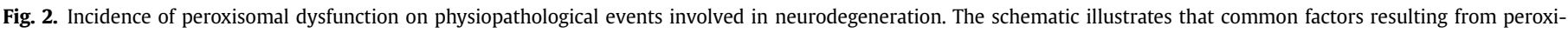

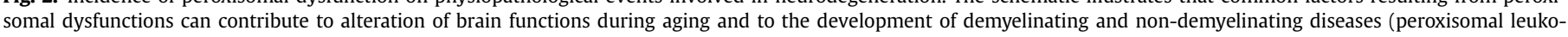

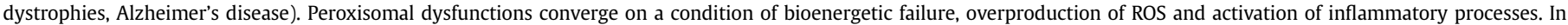

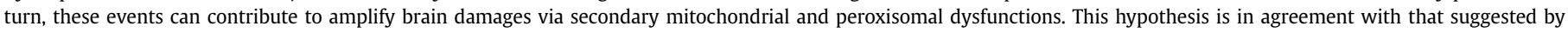
Galea et al. [48]. 
therapeutic target in the context of age-related diseases associated with oxidative stress [62]. Interestingly, the efficiency of the mitochondrial redox balance, a required condition to counteract aging processes, is perturbed in catalase-deficient cells and upon generation of excess ROS inside peroxisomes [51]. In addition, peroxisome life cycle may also be implicated in cellular aging. After peroxisome division, heterogeneous population of peroxisomes is observed in cells. Young and vital nascent organelles are present as well as old mature ones in which dysfunctional components accumulate due to damages caused by products of peroxisomal metabolism [66]. These old peroxisomes are eliminated by autophagy to maintain organelle homeostasis and to prevent accumulation of damaged peroxisomal compounds. Noteworthy, the deletion of the protein ATG1, required for autophagy, results in a significant increase in peroxisome numbers, accompanied with a decrease in cell viability in Hansenula polymorpha [67]. Moreover, in cells of advancing age, peroxisomal import of PTS1 proteins is reduced [68]. Collectively, a wide number of data support that peroxisomes are integrated into a system that governs cellular aging, and that peroxisomal dysfunctions might favor the occurrence of age-related diseases.

\subsection{Peroxisomes and Alzheimer's disease}

Alzheimer's disease (AD) is the most common form of dementia, characterized by progressive neurodegeneration, particularly affecting the hippocampal formation [69]. Impairment of cognitive and memory functions associated with accumulation of intracellular neurofibrillary tangles (NFT), extracellular amyloid $\beta(A \beta)$ peptide plaques and synaptic loss are the main important characteristic of this disease [70]. Based on the decline of peroxisomal activities with age [71], peroxisomal dysfunctions associated with aging and dementia including decreased catalase activity [72], lower plasmalogen synthesis [73], and increased sensitivity to cell death, especially necrosis $[74,75]$, might also favor neurodegenerative diseases such as $\mathrm{AD}[76,77]$.

The first evidence of the potential role of peroxisomes in the development of $\mathrm{AD}$ was established on primary rat hippocampal neuron cultures. In these cells, peroxisomal proliferation, induced by $\mathrm{Wy}-14.463$, which is a potent peroxisome proliferator-activated receptor $\alpha$ agonist, was shown to protect against cell death induced by the $A \beta$ peptide [78]. Moreover, on the Tg2576 mouse model of $A D$, significant peroxisome alterations were observed in the absence of apparent neuroanatomical or cytological signs of the disease [79]. In a transgenic mouse model, harboring the human Swedish familial AD mutation, the expression of ABCD3 and ACOX1 was found induced, possibly reflecting the need for efficient fatty acid $\beta$-oxidation, as a compensatory response to mitochondrial dysfunctions [80]. In rat, impaired peroxisome activity triggered by thioridazine also favors increased amyloid precursor protein (APP) and $\beta$-secretase (BACE1) expression and $A \beta$ generation at least partially by enhancing the VLCFA concentration [81].

In addition, substantial peroxisome-related alterations, which may contribute to the progression of AD pathology, were reported in humans. Several studies described a reduction of ethanolamine plasmalogen and choline plasmalogen levels in brain regions and erythrocyte of $\mathrm{AD}$ patients; this reduction was positively correlated with disease severity $[82,83]$. Moreover, based on the neuropathological Braak staging for $A D$, in stage $\mathrm{V}-\mathrm{VI}$ patients, increased levels of C22:0 and VLCFA (C24:0, C26:0), which are specific substrates of peroxisomal $\beta$-oxidation, a decrease of plasmalogens, an increased peroxisome volume density in neuronal cells and a decreased peroxisome number in neurites in $\mathrm{AD}$ brain were reported [84]. A reduction of DHA level was also observed in brain and liver of $\mathrm{AD}$ patients [85].
These results strengthen the hypothesis of the involvement of peroxisomal dysfunctions in the physiopathology of AD. Altogether, these data support a potential role of peroxisomes in neurodegeneration (Fig. 2).

\section{References}

[1] M. Islinger, S. Grille, H.D. Fahimi, M. Schrader, The peroxisome: an update on mysteries, Histochem. Cell Biol. 137 (2012) 547-574.

[2] N. Troffer-Charlier, N. Doerflinger, E. Metzger, F. Fouquet, J.L. Mandel, P. Aubourg, Mirror expression of adrenoleukodystrophy and adrenoleukodystrophy related genes in mouse tissues and human cell lines, Eur. J. Cell. Biol. 75 (1998) 254-264.

[3] M. Schrader, M. Thiemann, H.D. Fahimi, Peroxisomal motility and interaction with microtubules, Microsc. Res. Tech. 61 (2003) 171-178.

[4] J. Mathur, N. Mathur, M. Hulskamp, Simultaneous visualization of peroxisomes and cytoskeletal elements reveals actin and not microtubule-based peroxisome motility in plants, Plant Physiol. 128 (2002) 1031-1045.

[5] M. Fransen, M. Nordgren, B. Wang, O. Apanasets, Role of peroxisomes in ROS/ RNS-metabolism: implications for human disease, Biochim. Biophys. Acta 1822 (2012) 1363-1373.

[6] R.J. Wanders, H.R. Waterham, Biochemistry of mammalian peroxisomes revisited, Annu. Rev. Biochem. 75 (2006) 295-332.

[7] R.S. Chapkin, W. Kim, J.R. Lupton, D.N. McMurray, Dietary docosahexaenoic and eicosapentaenoic acid: emerging mediators of inflammation, Prostaglandins Leukot. Essent. Fatty Acids 81 (2009) 187-191.

[8] S. Ferdinandusse, S. Denis, P.A. Mooijer, Z. Zhang, J.K. Reddy, A.A. Spector, R.J. Wanders, Identification of the peroxisomal beta-oxidation enzymes involved in the biosynthesis of docosahexaenoic acid, J. Lipid Res. 42 (2001) 1987-1995.

[9] N. Salem Jr., B. Litman, H.Y. Kim, K. Gawrisch, Mechanisms of action of docosahexaenoic acid in the nervous system, Lipids 36 (2001) 945-959.

[10] A.A. Farooqui, n-3 fatty acid-derived lipid mediators in the brain: new weapons against oxidative stress and inflammation, Curr. Med. Chem. 19 (2012) 532-543.

[11] A.A. Farooqui, L.A. Horrocks, T. Farooqui, Modulation of inflammation in brain: a matter of fat, J. Neurochem. 101 (2007) 577-599.

[12] S.I. Rapoport, M. Igarashi, F. Gao, Quantitative contributions of diet and live synthesis to docosahexaenoic acid homeostasis, Prostaglandins Leukot. Essent. Fatty Acids 82 (2010) 273-276.

[13] P. Brites, H.R. Waterham, R.J. Wanders, Functions and biosynthesis of plasmalogens in health and disease, Biochim. Biophys. Acta 1636 (2004) 219-231.

[14] H. Hayashi, M. Hara, 1-Alkenyl group of ethanolamine plasmalogen derives mainly from de novo-synthesized fatty alcohol within peroxisomes, but not extraperoxisomal fatty alcohol or fatty acid, J. Biochem. 121 (1997) 978-983.

[15] N.E. Braverman, A.B. Moser, Functions of plasmalogen lipids in health and disease, Biochim. Biophys. Acta 1822 (2012) 1442-1452.

[16] P.A. Loughran, D.B. Stolz, S.R. Barrick, D.S. Wheeler, P.A. Friedman, R.A. Rachubinski, S.C. Watkins, T.R. Billiar, PEX7 and EBP50 target iNOS to the peroxisome in hepatocytes, Nitric Oxide 31 (2013) 9-19.

[17] L.A. Del Rio, Peroxisomes as a cellular source of reactive nitrogen species signal molecules, Arch. Biochem. Biophys. 506 (2011) 1-11.

[18] H.R. Waterham, M.S. Ebberink, Genetics and molecular basis of human peroxisome biogenesis disorders, Biochim. Biophys. Acta 1822 (2012) $1430-1441$

[19] S.J. Steinberg, G. Dodt, G.V. Raymond, N.E. Braverman, A.B. Moser H.W. Moser, Peroxisome biogenesis disorders, Biochim. Biophys. Acta 1763 (2006) 1733-1748.

[20] R. Wilson, J.R. Sargent, Lipid and fatty acid composition of brain tissue from adrenoleukodystrophy patients, J. Neurochem. 61 (1993) 290-297.

[21] F. Geillon, D. Trompier, C. Gondcaille, G. Lizard, S. Savary, Peroxisomal ABC transporters and X-linked adrenoleukodystrophy, Med. Sci. (Paris) 28 (2012) 1087-1094.

[22] C. Wiesinger, M. Kunze, G. Regelsberger, S. Forss-Petter, J. Berger, Impaired very long-chain acyl-CoA beta-oxidation in human X-linked adrenoleukodystrophy fibroblasts is a direct consequence of ABCD1 transporter dysfunction, J. Biol. Chem. 288 (2013) 19269-19279.

[23] J.M. Powers, H.W. Moser, Peroxisomal disorders: genotype, phenotype, major neuropathologic lesions, and pathogenesis, Brain Pathol. 8 (1998) 101-120.

[24] P.L. Faust, D. Banka, R. Siriratsivawong, V.G. Ng. T.M. Wikander, Peroxisome biogenesis disorders: the role of peroxisomes and metabolic dysfunction in developing brain, J. Inherit. Metab. Dis. 28 (2005) 369-383.

[25] S. Ferdinandusse, S. Denis, P.A. Mooyer, C. Dekker, M. Duran, R.J. SooraniLunsing, E. Boltshauser, A. Macaya, J. Gartner, C.B. Majoie, P.G. Barth, R.J. Wanders, B.T. Poll-The, Clinical and biochemical spectrum of D-bifunctional protein deficiency, Ann. Neurol. 59 (2006) 92-104.

[26] W.E. Kaufmann, C. Theda, S. Naidu, P.A. Watkins, A.B. Moser, H.W. Moser, Neuronal migration abnormality in peroxisomal bifunctional enzyme defect Ann. Neurol. 39 (1996) 268-271.

[27] R.I. Kelley, N.S. Datta, W.B. Dobyns, A.K. Hajra, A.B. Moser, M.J. Noetzel, E.H. Zackai, H.W. Moser, Neonatal adrenoleukodystrophy: new cases, biochemical studies, and differentiation from Zellweger and related peroxisomal polydystrophy syndromes, Am. J. Med. Genet. 23 (1986) 869-901. 
[28] A. Janssen, P. Gressens, M. Grabenbauer, E. Baumgart, A. Schad, I. Vanhorebeek, A. Brouwers, P.E. Declercq, D. Fahimi, P. Evrard, L. Schoonjans, D. Collen, P. Carmeliet, G. Mannaerts, P. Van Veldhoven, M. Baes, Neuronal migration depends on intact peroxisomal function in brain and in extraneuronal tissues, J. Neurosci. 23 (2003) 9732-9741.

[29] M. Baes, P. Aubourg, Peroxisomes, myelination, and axonal integrity in the CNS, Neuroscientist 15 (2009) 367-379.

[30] A.J. Barkovich, W.W. Peck, MR of Zellweger syndrome, AJNR Am. J. Neuroradiol. 18 (1997) 1163-1170.

[31] P.G. Barth, C.B. Majoie, J. Gootjes, R.J. Wanders, H.R. Waterham, M.S. van der Knaap, J.B. de Klerk, J. Smeitink, B.T. Poll-The, Neuroimaging of peroxisome biogenesis disorders (Zellweger spectrum) with prolonged survival, Neurology 62 (2004) 439-444.

[32] A.M. Bams-Mengerink, C.B. Majoie, M. Duran, R.J. Wanders, J. Van Hove C.D. Scheurer, P.G. Barth, B.T. Poll-The, MRI of the brain and cervical spinal cord in rhizomelic chondrodysplasia punctata, Neurology 66 (2006) 798-803.

[33] S. Ferdinandusse, M.S. Ylianttila, J. Gloerich, M.K. Koski, W. Oostheim, H.R. Waterham, J.K. Hiltunen, R.J. Wanders, T. Glumoff, Mutational spectrum of D-bifunctional protein deficiency and structure-based genotype-phenotype analysis, Am. J. Hum. Genet. 78 (2006) 112-124.

[34] D. Trompier, S. Savary, X-linked Adrenoleukodystrophy, Morgan \& Claypool Life Sciences, vol. 2, 2013, pp. 1-134.

[35] R. Chrast, G. Saher, K.A. Nave, M.H. Verheijen, Lipid metabolism in myelinating glial cells: lessons from human inherited disorders and mouse models, J. Lipid Res. 52 (2011) 419-434.

[36] N. Baumann, D. Pham-Dinh, Biology of oligodendrocyte and myelin in the mammalian central nervous system, Physiol. Rev. 81 (2001) 871-927.

[37] J.K. Ho, H. Moser, Y. Kishimoto, J.A. Hamilton, Interactions of a very long chain fatty acid with model membranes and serum albumin. Implications for the pathogenesis of adrenoleukodystrophy, J. Clin. Invest. 96 (1995) 1455-1463.

[38] M. Baarine, P. Andreoletti, A. Athias, T. Nury, A. Zarrouk, K. Ragot, A. Vejux, J.M. Riedinger, Z. Kattan, G. Bessede, D. Trompier, S. Savary, M. CherkaouiMalki, G. Lizard, Evidence of oxidative stress in very long chain fatty acid treated oligodendrocytes and potentialization of ROS production using RNA interference-directed knockdown of ABCD1 and ACOX1 peroxisomal proteins, Neuroscience 213 (2012) 1-18.

[39] S. Hein, P. Schonfeld, S. Kahlert, G. Reiser, Toxic effects of X-linked adrenoleukodystrophy-associated, very long chain fatty acids on glial cells and neurons from rat hippocampus in culture, Hum. Mol. Genet. 17 (2008) $1750-1761$.

[40] A. Bottelbergs, S. Verheijden, L. Hulshagen, D.H. Gutmann, S. Goebbels, K.A. Nave, C. Kassmann, M. Baes, Axonal integrity in the absence of functional peroxisomes from projection neurons and astrocytes, Glia 58 (2010) 15321543.

[41] J.M. Powers, Y. Liu, A.B. Moser, H.W. Moser, The inflammatory myelinopathy of adreno-leukodystrophy: cells, effector molecules, and pathogenetic implications, J. Neuropathol. Exp. Neurol. 51 (1992) 630-643.

[42] M.C. McGuinness, D.E. Griffin, G.V. Raymond, C.A. Washington, H.W. Moser K.D. Smith, Tumor necrosis factor-alpha and X-linked adrenoleukodystrophy, J. Neuroimmunol. 61 (1995) 161-169.

[43] J.M. Powers, The pathology of peroxisomal disorders with pathogenetic considerations, J. Neuropathol. Exp. Neurol. 54 (1995) 710-719.

[44] A.S. Paintlia, A.G. Gilg, M. Khan, A.K. Singh, E. Barbosa, I. Singh, Correlation of very long chain fatty acid accumulation and inflammatory disease progression in childhood X-ALD: implications for potential therapies, Neurobiol. Dis. 14 (2003) 425-439.

[45] C.M. Kassmann, C. Lappe-Siefke, M. Baes, B. Brugger, A. Mildner, H.B. Werner, O. Natt, T. Michaelis, M. Prinz, J. Frahm, K.A. Nave, Axonal loss and neuroinflammation caused by peroxisome-deficient oligodendrocytes, Nat. Genet. 39 (2007) 969-976.

[46] A. Bottelbergs, S. Verheijden, P.P. Van Veldhoven, W. Just, R. Devos, M. Baes, Peroxisome deficiency but not the defect in ether lipid synthesis causes activation of the innate immune system and axonal loss in the central nervous system, J. Neuroinflamm. 9 (2012) 61

[47] F.D. Mast, J. Li, M.K. Virk, S.C. Hughes, A.J. Simmonds, R.A. Rachubinski, A Drosophila model for the Zellweger spectrum of peroxisome biogenesis disorders, Dis. Model Mech. 4 (2011) 659-672.

[48] E. Galea, N. Launay, M. Portero-Otin, M. Ruiz, R. Pamplona, P. Aubourg, I. Ferrer, A. Pujol, Oxidative stress underlying axonal degeneration in adrenoleukodystrophy: a paradigm for multifactorial neurodegenerative diseases? Biochim. Biophys. Acta 1822 (2012) 1475-1488.

[49] M. Khan, J. Singh, A.G. Gilg, T. Uto, I. Singh, Very long-chain fatty acid accumulation causes lipotoxic response via 5-lipoxygenase in cerebral adrenoleukodystrophy, J. Lipid Res. 51 (2010) 1685-1695.

[50] I. Singh, A. Pujol, Pathomechanisms underlying X-adrenoleukodystrophy: a three-hit hypothesis, Brain Pathol. 20 (2010) 838-844.

[51] O. Ivashchenko, P.P. Van Veldhoven, C. Brees, Y.S. Ho, S.R. Terlecky, M. Fransen, Intraperoxisomal redox balance in mammalian cells: oxidative stress and interorganellar cross-talk, Mol. Biol. Cell 22 (2011) 1440-1451.

[52] J.M. Powers, Z. Pei, A.K. Heinzer, R. Deering, A.B. Moser, H.W. Moser, P.A. Watkins, K.D. Smith, Adreno-leukodystrophy: oxidative stress of mice and men, J. Neuropathol. Exp. Neurol. 64 (2005) 1067-1079.

[53] B. Ahlemeyer, M. Gottwald, E. Baumgart-Vogt, Deletion of a single allele of the Pex11beta gene is sufficient to cause oxidative stress, delayed differentiation and neuronal death in mouse brain, Dis. Model Mech. 5 (2012) 125-140.
[54] C.C. Muller, T.H. Nguyen, B. Ahlemeyer, M. Meshram, N. Santrampurwala, S. Cao, P. Sharp, P.B. Fietz, E. Baumgart-Vogt, D.I. Crane, PEX13 deficiency in mouse brain as a model of Zellweger syndrome: abnormal cerebellum formation, reactive gliosis and oxidative stress, Dis. Model Mech. 4 (2011) 104-119.

[55] M. Baarine, K. Ragot, A. Athias, T. Nury, Z. Kattan, E.C. Genin, P. Andreoletti, F. Menetrier, J.M. Riedinger, M. Bardou, G. Lizard, Incidence of Abcd1 level on the induction of cell death and organelle dysfunctions triggered by very long chain fatty acids and TNF-alpha on oligodendrocytes and astrocytes, Neurotoxicology 33 (2012) 212-228.

[56] I. Singh, K. Pahan, M. Khan, A.K. Singh, Cytokine-mediated induction of ceramide production is redox-sensitive. Implications to proinflammatory cytokine-mediated apoptosis in demyelinating diseases, J. Biol. Chem. 273 (1998) 20354-20362.

[57] V. Feigenbaum, A. Gelot, P. Casanova, C. Daumas-Duport, P. Aubourg, M. Dubois-Dalcq, Apoptosis in the central nervous system of cerebral adrenoleukodystrophy patients, Neurobiol. Dis. 7 (2000) 600-612.

[58] F.S. Eichler, J.Q. Ren, M. Cossoy, A.M. Rietsch, S. Nagpal, A.B. Moser, M.P. Frosch, R.M. Ransohoff, Is microglial apoptosis an early pathogenic change in cerebral X-linked adrenoleukodystrophy? Ann. Neurol. 63 (2008) $729-742$.

[59] M. Ito, B.M. Blumberg, D.J. Mock, A.D. Goodman, A.B. Moser, H.W. Moser, K.D. Smith, J.M. Powers, Potential environmental and host participants in the early white matter lesion of adreno-leukodystrophy: morphologic evidence for CD8 cytotoxic T cells, cytolysis of oligodendrocytes, and CD1-mediated lipid antigen presentation, J. Neuropathol. Exp. Neurol. 60 (2001) 1004-1019.

[60] E. Kahn, M. Baarine, A. Dauphin, K. Ragot, N. Tissot, A. Seguin, F. Menetrier, Z. Kattan, C.M. Bachelet, F. Frouin, G. Lizard, Impact of 7-ketocholesterol and very long chain fatty acids on oligodendrocyte lipid membrane organization: evaluation via LAURDAN and FAMIS spectral image analysis, Cytometry A 79 (2011) 293-305.

[61] A. Zarrouk, A. Vejux, T. Nury, H.I. El Hajj, M. Haddad, M. Cherkaoui-Malki, J.M. Riedinger, M. Hammami, G. Lizard, Induction of mitochondrial changes associated with oxidative stress on very long chain fatty acids (C22:0, C24:0, or C26:0)-treated human neuronal cells (SK-NB-E), Oxid. Med. Cell. Longev. 2012 (2012) 623257.

[62] C.R. Giordano, S.R. Terlecky, Peroxisomes, cell senescence, and rates of aging, Biochim. Biophys. Acta 1822 (2012) 1358-1362.

[63] L. Goth, J.W. Eaton, Hereditary catalase deficiencies and increased risk of diabetes, Lancet 356 (2000) 1820-1821.

[64] C.S. Wood, J.I. Koepke, H. Teng, K.K. Boucher, S. Katz, P. Chang, L.J. Terlecky, I. Papanayotou, P.A. Walton, S.R. Terlecky, Hypocatalasemic fibroblasts accumulate hydrogen peroxide and display age-associated pathologies, Traffic 7 (2006) 97-107.

[65] J.I. Koepke, C.S. Wood, L.J. Terlecky, P.A. Walton, S.R. Terlecky, Progeric effects of catalase inactivation in human cells, Toxicol. Appl. Pharmacol. 232 (2008) 99-108.

[66] A. Beach, M.T. Burstein, V.R. Richard, A. Leonov, S. Levy, V.I. Titorenko, Integration of peroxisomes into an endomembrane system that governs cellular aging, Front. Physiol. 3 (2012) 283.

[67] E.B. Aksam, A. Koek, J.A. Kiel, S. Jourdan, M. Veenhuis, I.J. van der Klei, A peroxisomal lon protease and peroxisome degradation by autophagy play key roles in vitality of Hansenula polymorpha cells, Autophagy 3 (2007) 96-105.

[68] J.E. Legakis, J.I. Koepke, C. Jedeszko, F. Barlaskar, L.J. Terlecky, H.J. Edwards, P.A. Walton, S.R. Terlecky, Peroxisome senescence in human fibroblasts, Mol. Biol. Cell 13 (2002) 4243-4255.

[69] M.W. Marlatt, P.J. Lucassen, Neurogenesis and Alzheimer's disease: biology and pathophysiology in mice and men, Curr. Alzheimer Res. 7 (2010) 113-125.

[70] H.W. Querfurth, F.M. LaFerla, Alzheimer's disease, N. Engl. J. Med. 362 (2010) 329-344.

[71] V.I. Titorenko, S.R. Terlecky, Peroxisome metabolism and cellular aging, Traffic 12 (2011) 252-259.

[72] D.L. Marcus, C. Thomas, C. Rodriguez, K. Simberkoff, J.S. Tsai, J.A. Strafaci, M.L. Freedman, Increased peroxidation and reduced antioxidant enzyme activity in Alzheimer's disease, Exp. Neurol. 150 (1998) 40-44.

[73] D.B. Goodenowe, L.L. Cook, J. Liu, Y. Lu, D.A. Jayasinghe, P.W. Ahiahonu, D. Heath, Y. Yamazaki, J. Flax, K.F. Krenitsky, D.L. Sparks, A. Lerner, R.P. Friedland, T. Kudo, K. Kamino, T. Morihara, M. Takeda, P.L. Wood, Peripheral ethanolamine plasmalogen deficiency: a logical causative factor in Alzheimer's disease and dementia, J. Lipid Res. 48 (2007) 2485-2498.

[74] S. Manivannan, C.Q. Scheckhuber, M. Veenhuis, I.J. van der Klei, The impact of peroxisomes on cellular aging and death, Front. Oncol. 2 (2012) 50.

[75] J. Lopez-Erauskin, I. Ferrer, E. Galea, A. Pujol, Cyclophilin D as a potential target for antioxidants in neurodegeneration: the X-ALD case, Biol. Chem. 394 (2013) 621-629.

[76] S.R. Terlecky, J.I. Koepke, P.A. Walton, Peroxisomes and aging, Biochim. Biophys. Acta 1763 (2006) 1749-1754.

[77] G. Lizard, O. Rouaud, J. Demarquoy, M. Cherkaoui-Malki, L. Iuliano, Potential roles of peroxisomes in Alzheimer's disease and in dementia of the Alzheimer's type, J. Alzheimers Dis. 29 (2012) 241-254.

[78] M.J. Santos, R.A. Quintanilla, A. Toro, R. Grandy, M.C. Dinamarca, J.A. Godoy, N.C. Inestrosa, Peroxisomal proliferation protects from beta-amyloid neurodegeneration, J. Biol. Chem. 280 (2005) 41057-41068.

[79] A. Cimini, S. Moreno, M. D’Amelio, L. Cristiano, B. D’Angelo, S. Falone, E. Benedetti, P. Carrara, F. Fanelli, F. Cecconi, F. Amicarelli, M.P. Ceru, Early 
biochemical and morphological modifications in the brain of a transgenic mouse model of Alzheimer's disease: a role for peroxisomes, J. Alzheimers Dis. (2009).

[80] F. Fanelli, S. Sepe, M. D’Amelio, C. Bernardi, L. Cristiano, A. Cimini, F. Cecconi, M.P. Ceru, S. Moreno, Age-dependent roles of peroxisomes in the hippocampus of a transgenic mouse model of Alzheimer's disease, Mol. Neurodegener. 8 (2013) 8.

[81] R. Shi, Y. Zhang, Y. Shi, S. Shi, L. Jiang, Inhibition of peroxisomal beta-oxidation by thioridazine increases the amount of VLCFAs and Abeta generation in the rat brain, Neurosci. Lett. 528 (2012) 6-10.

[82] M. Igarashi, K. Ma, F. Gao, H.W. Kim, S.I. Rapoport, J.S. Rao, Disturbed choline plasmalogen and phospholipid fatty acid concentrations in Alzheimer's disease prefrontal cortex, J. Alzheimers Dis. 24 (2011) 507-517.
[83] P.L. Wood, R. Mankidy, S. Ritchie, D. Heath, J.A. Wood, J. Flax, D.B. Goodenowe Circulating plasmalogen levels and Alzheimer disease assessment scalecognitive scores in Alzheimer patients, J. Psychiatry Neurosci. 35 (2010) $59-62$.

[84] J. Kou, G.G. Kovacs, R. Hoftberger, W. Kulik, A. Brodde, S. Forss-Petter, S. Honigschnabl, A. Gleiss, B. Brugger, R. Wanders, W. Just, H. Budka, S. Jungwirth, P. Fischer, J. Berger, Peroxisomal alterations in Alzheimer's disease, Acta Neuropathol. 122 (2011) 271-283.

[85] G. Astarita, K.M. Jung, N.C. Berchtold, V.Q Nguyen, D.L. Gillen, E. Head, C.W. Cotman, D. Piomelli, Deficient liver biosynthesis of docosahexaenoic acid correlates with cognitive impairment in Alzheimer's disease, PLoS One 5 (2010) e12538. 